March 1999

UM-P-99/07

KIAS-P99023

UDELHP-99/101

\title{
Closing the Neutrinoless Double Beta Decay Window into Violations of the Equivalence Principle and/or Lorentz Invariance
}

\author{
A. Halprin $(b, c)$ and R.R. Volkas (a) \\ (a) School of Physics, Research Centre for High Energy Physics, The University of Melbourne, \\ Parkville 3052 Australia \\ (b) Department of Physics and Astronomy, The University of Delaware \\ Newark, DE 19716, USA \\ (c) Korean Institute for Advanced Study \\ 207-43 Cheongryangri-dong, Dongdaemun-gu, Seoul 130-012, Korea \\ (halprin@udel.edu,r.volkas@physics.unimelb.edu.au)
}

\begin{abstract}
We have examined Lorentz invariance and equivalence principle violations in the neutrino sector as manifested in neutrinoless double beta decay. We conclude that this rare decay cannot provide a useful view of these exotic processes.
\end{abstract}




\section{INTRODUCTION}

During the past few years, neutrino oscillations have been used to explore exotic properties of neutrinos such as possible violations of Lorentz invariance (VLI) [1 3] and/or violations of the equivalence principle (VEP) [ [4 9]. Since neutrinolesss double beta decay has served as a window into neutrino masses for the last two decades [10 12], it is natural to enquire if this rare decay can tell us anything about VLI and VEP processes.

We begin with the observation that the properties of neutrinos enter into the neutrino exchange diagram for the neutrinoless double beta decay amplitude (without right handed currents) in the form of the factor [13]

$$
A^{\nu}=\left(1-\gamma_{5}\right) P\left(1-\gamma_{5}\right)
$$

where $\mathrm{P}$ is a linear combination of the propagators for each of the Majorana neutrino fields that constitute the $\nu_{e}$ field,

$$
P=U_{e a}^{2} P_{a}
$$

The $U_{e a}$ are elements of the unitary matrix connecting mass and weak eigenstate neutrinos. In the absence of external fields, and in the absence of Lorentz invariance violation, the Majorana fields have definite masses, $m_{a}$, and all neutrinos have the same limiting velocity, c. In that case the $P_{a}$ are, of course, given by

$$
P_{a}=\left(\gamma^{0} E-\gamma^{k} p_{k} c+m_{a} c^{2}\right)^{-1} .
$$

\section{MODIFICATIONS DUE TO VIOLATION OF LORENTZ INVARIANCE.}

In the Lorentz invariance violating scheme of Coleman and Glashow, the limiting velocity of each neutrino may be distinct, so that $c \rightarrow c_{a}$. Our conclusions are unaltered by the introduction of distinct mass and velocity bases, and for the sake of clarity such a complication will be ignored. To first order in $m_{a}$, the VLI modified $A^{\nu}$ is then given by

$$
A_{V L I}^{\nu} \simeq-\frac{2 U_{e a}^{2}\left(1-\gamma_{5}\right) m_{a} c_{a}^{2}}{E^{2}-\left(p c_{a}\right)^{2}}
$$

where the chirality factors have been used to eliminate contributions from factors of $E \gamma^{0}$ or $\gamma^{k} p_{k}$ in the numerator.

For neutrinoless double beta decay in nuclei we usually make the approximation of ignoring nuclear recoil, so the energy of the exchanged neutrino is set equal to zero, that is $E=0$. With this standard approximation,

$$
A_{V L I}^{\nu} \simeq 2 U_{e a}^{2}\left(1-\gamma_{5}\right) m_{a} / p^{2} .
$$

Since this expession is independent of limiting velocities, we conclude that VLI cannot enter into neutrinoless double beta decay in any significant way. 


\section{MODIFICATIONS DUE TO VIOLATION OF THE EQUIVALENCE PRINCIPLE.}

Following the formalism of Ref. [6], the Dirac equation governing neutrino $a$ is modified by the presence of an external gravitational field which couples to neutrinos with strength $f_{a}$ relative to the usual universal Newtonian coupling. For the sake of clarity, we take the mass and gravitational coupling bases to be the same. In the presence of a constant Newtonian potential, $\Phi$, the neutrino propagator becomes

$$
P_{a}=\left[\left(1+f_{a} \Phi\right) E \gamma^{0}-\left(1-f_{a} \Phi\right) \gamma^{k} p_{k}+m_{a}\right]^{-1}
$$

where we have set the common limiting vacuum (i.e. $\Phi=0$ ) velocity equal to 1 .

To first order in $m_{a}$ we then have, making use of the chirality factors as before,

$$
A_{V E P}^{\nu} \simeq-\frac{2 U_{e a}^{2}\left(1-\gamma_{5}\right) m_{a}}{\left(1+f_{a} \Phi\right)^{2} E^{2}-\left(1-f_{a} \Phi\right)^{2} p^{2}} .
$$

Making the zero recoil approximation as above and retaining only to first order in $\Phi$ for consistency, we then have

$$
A_{V E P}^{\nu} \simeq 2 U_{e a}^{2}\left(1-\gamma_{5}\right) m_{a}\left(1+2 f_{a} \Phi\right) / p^{2} .
$$

The expression above includes only the modification to the neutrino propagator due to the presence of $\Phi$. To this we must also add modifications to the $\mathrm{W}$ - boson, quark and electron lines due to $\Phi$. Assuming that only neutrinos have anomalous gravitational couplings, restoration of gravitational gauge invariance in the limit that all $f_{a}$ are equal guarantees that the final $\Phi$-dependent contribution depends only upon $\Delta f_{a}=f_{a}-f_{0}$, where $f_{0}=1$ in Einsteinian gravity. The $\Phi$-dependent contribution to the total neutrinoless double beta decay rate will then be proportional to $U_{e a}^{2} m_{a} \Delta f_{a}$. Thus, the VEP effect is proportional to both $m_{a}$ and $\Delta f_{a}$ and is therefore extremely small.

\section{CONCLUSIONS}

We have examined the modifications to the usual neutrino exchange diagram for the neutrinoless double beta decay amplitude arising from violations of Lorentz invariance and/or the equivalence principle in the neutrino sector. We find that the VLI parameters disappear from the decay amplitude in the usual zero recoil approximation and that the VEP parameters enter the amplitude only in combination with with neutrino mass factors. We therefore conclude that neutrinolesss double beta decay cannot provide a significant window into VLI and VEP neutrino processes. This result appears to contradict the conclusions of a recent paper [14].

\section{ACKNOWLEDGMENTS}

A.H. would like to thank the particle theory group at The University of Melbourne and the Korean Institute for Advanced Study for their hospitality during a portion of this work. 
He also thanks C.N. Leung for discussions during the early stages of this work. This work was supported in part by the US Department of Energy grant DE-FG02-84ER40163. R.R.V. was supported by the Australian Research Council. 


\section{REFERENCES}

[1] S. Coleman and S.L. Glashow, preprint hep-ph/9812418 (January 1999).

[2] S. Coleman and S.L. Glashow, Phys. Lett. B405, 249 (1997)

[3] S.L. Glashow, A. Halprin, P.I. Krastev, C.N. Leung, and J. Pantaleone, Phys. Rev. D56, 2433 (1997).

[4] R. Foot, C.N. Leung and O. Yasuda, Phys. Lett. B443, 185 (1998).

[5] A. Halprin, C.N. Leung, and J. Pantaleone, Phys. Rev. D53, 5365 (1996). The symbol $\gamma$ of this reference has been replaced by $f_{a}$.

[6] K. Iida, H. Minakata and O. Yasuda, Mod. Phys. Lett A8, 1037 (1993).

[7] A. Halprin and C.N. Leung, Phys. Rev. Lett. 67, 1833 (1991).

[8] M. Gasperini, Phys. Rev. D38, 2635 (1988);ibid. D39, 3606 (1989).

[9] M. Gasparini, Phys. Rev. Lett. 62, 1945 (1989)

[10] A. Halprin, P. Minkowski, H. Primakoff and S.P. Rosen, Phys. Rev D13, 2567 (1976).

[11] M. Doi, T. Kotani and E. Takasugi, Prog. Theor. Phys. Suppl. 83, 1 (1985).

[12] For a recent review, see R.N. Mohapatra, Proceedings of Neutrino98, Takayama, Japan, June 1998.

[13] A very thorough discussion of this point can be found in B. Kayser, F. Gibrat-Debu and F. Perrier, The Physics of Massive Neutrinos, (World Scientific, Singapore, 1989)

[14] H.V. Klapdor-Kleingrothaus, H. Päs, U. Sarkar, preprint hep-ph/9809396 (September 1998). 